\title{
Modeling Multiwavelength Blazar Spectra using a Particle Transport Equation
}

\author{
Tiffany R. Lewis ${ }^{1}$ \\ George Mason University \\ 4400 University Dr. MSN: 3F3, Fairfax, VA 22030, USA \\ E-mail: tlewis13.gmu@gmail.com \\ Justin D. Finke \\ United States Naval Research Laboratory \\ 4555 Overlook Ave, SW, Washington, DC 20375, USA \\ E-mail: justin.finke@nrl.navy.mil
}

\section{Peter A. Becker}

George Mason University

4400 University Dr. MSN: 3F3, Fairfax, VA 22030, USA

E-mail: pbeckeregmu. edu

Blazars are luminous extragalactic sources across the entire electromagnetic spectrum, but the spectral formation mechanisms in these sources are not well understood. We have developed a new theoretical model for simulating blazar spectra in which we numerically integrate the electron transport equation to generate the electron number distribution with respect to energy. Our transport model considers shock acceleration, adiabatic expansion, stochastic acceleration due to MHD waves, Bohm diffusive particle escape, synchrotron radiation, and Compton radiation. We implement the full Compton cross-section for electron interactions with photons from dust and 26 lines from the broad line region, each considered individually, without additional free parameters. We use the solution for the electron distribution to calculate multi-wavelength SED spectra for 3C 279. This new, self-consistent model provides an unprecedented view into the jet physics at play in this source, especially the relative strength of the shock and stochastic acceleration components and location of the emitting region. We show that our new Compton + synchrotron blazar model is the first to successfully fit the Fermi-LAT gamma-ray data for this source based on a self-consistent, first-principles physical calculation.

7th Fermi Symposium 2017

15-20 October 2017

Garmisch-Partenkirchen, Germany

\footnotetext{
${ }^{1}$ Speaker

(C) Copyright owned by the author(s) under the terms of the Creative Commons 